\title{
Quantum Nondemolition Squeezing of a Nanomechanical Resonator
}

\author{
Rusko Ruskov, Keith Schwab, and Alexander N. Korotkov
}

\begin{abstract}
We show that the nanoresonator position can be squeezed significantly below the ground state level by measuring the nanoresonator with a quantum point contact or a single-electron transistor and applying a periodic voltage across the detector. The mechanism of squeezing is basically a generalization of quantum nondemolition measurement of an oscillator to the case of continuous measurement by a weakly coupled detector. The quantum feedback is necessary to prevent the "heating" due to measurement back-action. We also discuss a procedure of experimental verification of the squeezed state.
\end{abstract}

Index Terms-Nano-electromechanical systems (NEMS), quantum feedback, squeezing.

\section{INTRODUCTION}

$\mathbf{Q}$ UANTUM nondemolition (QND) measurements [1]-[4] were proposed as a way to overcome the so-called standard quantum limit for the measurement sensitivity, which arises due to quantum back-action of a detector. The general idea of a QND measurement is to avoid measuring (or obtaining any information on) the magnitude conjugated to the magnitude of interest and, therefore, to avoid the corresponding back-action. An important implementation of this idea is the "stroboscopic" measurement of an oscillator position [2], [3]. Suppose the position $x_{1}$ is measured (instantaneously) with a finite precision $\Delta x$, which necessarily disturbs the momentum according to the Heisenberg uncertainty principle $\Delta p \geq \hbar / 2 \Delta x$. Normally this momentum change would affect the result of the next position measurement $x_{2}$ and would limit the accuracy for the position difference $x_{2}-x_{1}$, leading to the standard quantum limit for this magnitude. However, if the second measurement is performed exactly one oscillation period after the first one, the oscillator returns to its initial state and, therefore, the momentum change does not affect the accuracy of the $x_{2}-x_{1}$ measurement. Such stroboscopic measurement gives no information related to the momentum, and this is exactly the reason why the effect of quantum back-action is avoided [1]-[4].

In terms of applications, the QND measurements have been mainly discussed in relation to measurement of very weak classical forces, in particular, gravitational waves (see, e.g., [5] and

Manuscript received June 15, 2004; revised August 7, 2004. The work of R. Ruskov and A. N. Korotkov was supported by the National Security Agency and the Advanced Research and Development Activity under ARO Grant DAAD 19-01-1-0491. The work of K. Schwab was supported by the National Security Agency.

R. Ruskov and A. N. Korotkov are with the Department of Electrical Engineering, University of California, Riverside, CA 92521 USA.

K. Schwab is with the Laboratory for Physical Sciences, National Security Agency, College Park, MD 20740 USA.

Digital Object Identifier 10.1109/TNANO.2004.840171
[6]). Recently, the idea of QND measurements has been also applied to solid-state mesoscopic structures (see, e.g., [7] and [8]). Among other recent developments (the total number of papers on QND measurements is over 500), let us mention the experiment on atomic spin-squeezing using the QND measurement and real-time quantum feedback [9]. In this paper, we discuss squeezing of a nanomechanical resonator using the QND measurement and quantum feedback.

Recent advances in fabrication of nanomechanical resonators [10]-[14], [45]-[48] make possible the direct observation of their quantum behavior in the nearest future. For the resonator frequency $\omega_{0} / 2 \pi$ exceeding $1 \mathrm{GHz}$ [12], the condition $T<\hbar \omega_{0}$ (we use $k_{B}=1$ ) is satisfied at temperature $T$ below $\sim 50 \mathrm{mK}$. Even in the case $T \gg \hbar \omega_{0}$, the quantum behavior is in principle observable [1] when $T \tau / Q<\hbar / 2$, where $Q$ is the resonator quality factor and $\tau$ is the typical observation time. This condition can be well satisfied even for megahertz-range resonators for $\tau$ comparable to the oscillation period, i.e., if we can monitor the oscillations with the measurement bandwidth on the order of $\omega_{0}$, as in [14]. There is a rapid experimental progress in monitoring the oscillating position of a nanoresonator using a radio-frequency single-electron transistor (RF-SET) [13], [14] or quantum point contact (QPC) [15] (at present, the RF-SET seems to be much more efficient). In particular, the position measurement accuracy $\Delta x$ within the factor 5.8 from the standard quantum limit $\Delta x_{0}$ has been demonstrated [14]; here $\Delta x_{0}=\sqrt{\hbar / 2 m \omega_{0}}$ is the width (standard deviation) of the ground state of the oscillator with mass $m$. Measurement of the nanoresonator position by RF-SET or QPC has also received a significant theoretical attention [16]-[22], [49], [50]. The process of measurement transfers the energy from the detector to the nanoresonator leading to its "heating" [16], [17], [49], [50]. A possible way to prevent such heating is by using the quantum feedback control of the nanoresonator [22] (another idea for cooling has been proposed in [23]).

The quantum feedback of mesoscopic solid-state systems is a relatively new subject [24], [51], though in quantum optics the quantum feedback was proposed more than a decade ago [25] and has already been realized experimentally [9]. The feedback analyzed in [22] assumes continuous monitoring of the nanoresonator position with constant "strength" of measurement and allows cooling of the nanoresonator practically down to the ground state. However, it does not allow squeezing of the nanoresonator state (below $\Delta x_{0}$ ), which would be important, for example, for the ultrasensitive measurement of a force acting on the nanoresonator. (More correctly, squeezing in [22] is possible only in the unrealistic case of a strong coupling between the nanoresonator and detector.) 
In this paper, we analyze the case of the nanoresonator monitoring with the measurement strength modulated in time (for example, modulating the bias voltage of the QPC or RF-SET), basically applying the idea of stroboscopic QND measurements [2], [3] to the nanoresonator. We show that, in this case, even for a weak coupling with a detector, a significant squeezing of the nanoresonator state can be achieved when the modulation frequency $\omega$ is close to $2 \omega_{0} / n, n=1,2, \ldots$. The simple physical reason of this result is that the total Hamiltonian evolution during one period of resonator oscillation is exactly zero (unity operator); therefore, a repeated weak measurement at proper moments of time adds up to a strong measurement, which naturally produces a squeezed state. The difference from the QND measurement of [2] and [3] is that we consider continuous in time measurement and assume weak coupling between the nanoresonator and the detector. Therefore, exact matching with the oscillation period is impossible, which leads to additional heating due to measurement (above the level required by squeezing), so we need to use the quantum feedback to prevent this extra heating. We would also like to notice the difference between our proposal and squeezing of a nanoresonator proposed in [26], which is a scaled-down version of the classical thermomechanical noise squeezing [27] using the modulation of the resonator spring constant.

\section{SYSTEM AND MODEL}

For simplicity, we consider the nanoresonator measured by the low-transparency QPC (though our results are applicable to the RF-SET as well) and the system Hamiltonian is

$$
\mathcal{H}=\mathcal{H}_{0}+\mathcal{H}_{\text {det }}+\mathcal{H}_{\text {int }}+\mathcal{H}_{\text {fb }}
$$

where the first term describes the oscillator (nanoresonator)

$$
\mathcal{H}_{0}=\frac{\hat{p}^{2}}{2 m}+\frac{m \omega_{0}^{2} \hat{x}^{2}}{2}
$$

where $\hat{p}$ and $\hat{x}$ are the momentum and position operators, the last term

$$
\mathcal{H}_{\text {fb }}=-\mathcal{F} \hat{x}
$$

describes the feedback control of the nanoresonator by applying the force $\mathcal{F}(t)$, while $\mathcal{H}_{\text {det }}$ and $\mathcal{H}_{\text {int }}$ correspond to the detector and its interaction with the nanoresonator similar to [17], [28]

$$
\begin{aligned}
\mathcal{H}_{\mathrm{det}} & =\sum_{l} E_{l} a_{l}^{\dagger} a_{l}+\sum_{r} E_{r} a_{r}^{\dagger} a_{r}+\sum_{l, r}\left(M a_{l}^{\dagger} a_{r}+\text { H.c. }\right) \\
\mathcal{H}_{\mathrm{int}} & =\sum_{l, r}\left(\Delta M \hat{x} a_{l}^{\dagger} a_{r}+\text { H.c. }\right) .
\end{aligned}
$$

Here, $a_{l, r}^{\dagger}$ and $a_{l, r}$ are the creation and annihilation operators for two electrodes of the QPC, and, for simplicity, we assume no relative phase between the tunneling amplitudes $M$ and $\Delta M$ (taking this phase into account is simple [29]-[31], but makes the formalism significantly lengthier). For a given position state $|x\rangle$ of the oscillator, the average detector current is $I_{x}=2 \pi|M+\Delta M x|^{2} \rho_{l} \rho_{r} e^{2} V / \hbar$, where $V$ is the QPC voltage which may vary in time with frequency $\omega$ comparable to $\omega_{0}, e$ is the electron charge, and $\rho_{l, r}$ are the densities of states in the electrodes.

We assume a weak response of the detector, $\left|I_{x}-I_{x^{\prime}}\right| \ll$ $I_{x}+I_{x^{\prime}}$ and, therefore, the linear dependence of the detector current on the measured position is

$$
I_{x}=I_{0}+k x
$$

Also, we neglect the dependence on $x$ of the detector current spectral density $S_{I}$ which is assumed to be flat in the frequency range of interest. Because the voltage $V$ varies in time, $I_{0}, k$, $I_{x}$, and $S_{I}$ also depend on time, which will be taken into account explicitly in Section III. Notice that the white noise $S_{I}$ is an intrinsic detector noise, which is defined for a fixed voltage on a time scale much shorter than the time scale of voltage variations, while the long-time spectral density of the detector current is obviously affected by the voltage changes as well as by the oscillating signal from the nanoresonator.

Somewhat similar to the case of qubit measurement [32], [52], we define the dimensionless (time-dependent) coupling as

$$
\mathcal{C}=\frac{\hbar k^{2}}{S_{I} m \omega_{0}^{2}}
$$

which can also be expressed as $\mathcal{C}=4 / \omega_{0} \tau_{m}$, where $\tau_{m}=$ $2 S_{I} /\left(k \Delta x_{0}\right)^{2}$ is the "measurement" time which would be necessary to distinguish (with an SNR of 1) two position states separated by the ground state width $\Delta x_{0}=\sqrt{\hbar / 2 m \omega_{0}}$. We will mainly consider the case of weak coupling, $\mathcal{C} \ll 1$, which corresponds to a realistic experimental situation. As an example, $\mathcal{C}$ is of the order of $10^{-3}$ for the parameters of experiment [14].

To describe the dynamics of the quantum measurement process, we apply the quantum Bayesian approach [32], [52], developed initially for measurement of qubits by solid-state detectors (this approach is practically equivalent to the approach of quantum trajectories used, e.g., in [22], [25], [30], and [33]). The Bayesian formalism takes into account the information about the measured system evolution contained in the noisy detector output and, therefore, is able to describe quantum evolution in a particular realization of the measurement process (without ensemble averaging). The main statements of the Bayesian formalism are that the evolution of the diagonal matrix elements of the measured system density matrix is governed by the classical Bayes theorem (on conditional probability) and that the pure state of the measured system remains pure when measured by an ideal detector (QPC is an example of an ideal detector); a nonideal detector can be described as an ideal detector with extra noise sources. While using the quantum Bayesian approach, we need to use its usual assumptions [32], [52]; in particular, we assume that the internal dynamics of the detector is much faster than the oscillator dynamics (this requires $\left.\mathrm{eV} \gg \hbar \omega_{0}\right)$, and we assume a quasi-continuous detector current (which requires $I_{0} / e \gg \omega_{0}$ and even stronger inequality $\left.k \Delta x_{0} / e \gg \omega_{0}\right)$.

Applying the Bayesian approach to our system, we derive (the derivation will be presented elsewhere) the following equation 
for the evolution of oscillator density matrix $\rho$ in $x$-basis (in Stratonovich form):

$$
\begin{aligned}
\dot{\rho}\left(x, x^{\prime}\right)= & \frac{-i}{\hbar}\left[\mathcal{H}_{0}+\mathcal{H}_{f b}, \rho\right]_{x, x^{\prime}}+\rho\left(x, x^{\prime}\right) \frac{1}{S_{I}} \\
& \times\left\{I(t)\left(I_{x}+I_{x^{\prime}}-2\langle I\rangle\right)-\left(\frac{I_{x}^{2}+I_{x^{\prime}}^{2}}{2}-\left\langle I^{2}\right\rangle\right)\right\}
\end{aligned}
$$

where the first term is a usual evolution due to $\mathcal{H}_{0}+\mathcal{H}_{f b}$, while the second term describes the evolution due to measurement and, therefore, depends on the noisy detector current $I(t)$; we introduced notations $\langle I\rangle=\int I_{x} \rho(x, x) d x$ and $\left\langle I^{2}\right\rangle=\int I_{x}^{2} \rho(x, x) d x$. Notice that (4) actually does not require the current linearity (2). Also notice that (4) coincides with the similar equation for the case of an arbitrary number of entangled qubits measured by an ideal detector [31], if $x$ is replaced by the index corresponding to the state of qubits.

Equation (4) allows us to monitor the oscillator density matrix $\rho$ using the measurement record $I(t)$, while for simulations $I(t)$ may be replaced with

$$
I(t)=\langle I\rangle+\xi(t)
$$

where $\xi(t)$ is a white noise with spectral density $S_{I}$. (These equations are derived assuming a constant detector voltage; however, they can still be used when the voltage (or any other parameter) variation is much slower than internal dynamics of the detector, which involves time constants $\hbar / e V$ and $e /\langle I\rangle$.)

Translating (4) from Stratonovich into Ito form, using current linearity (2), and taking into account quantum efficiency $\eta$ of the detector [32], [52] (QPC is an ideal detector, $\eta=1$, while $\eta<1$ can be used for the RF-SET as a detector), we obtain

$$
\begin{aligned}
\dot{\rho}\left(x, x^{\prime}\right)= & \frac{-i}{\hbar}\left[\mathcal{H}_{0}+\mathcal{H}_{f b}, \rho\right]_{x, x^{\prime}}-\frac{k^{2}}{4 S_{I} \eta}\left(x-x^{\prime}\right)^{2} \rho\left(x, x^{\prime}\right) \\
& +\frac{k}{S_{I}}\left(x+x^{\prime}-2\langle x\rangle\right) \rho\left(x, x^{\prime}\right) \xi(t)
\end{aligned}
$$

where $\langle x\rangle=\int x \rho(x, x) d x$. Equation (6) is similar to equations derived in many publications (e.g., in [22] and [33]-[35]) for measurement of a mechanical oscillator. Averaging (6) over the measurement record $I(t)$ eliminates the last term of (6) [in Ito form, averaging over the noise $\xi(t)$ is equivalent to using $\xi(t)=0$ ] and leads to the ensemble averaged equation derived in even larger number of papers, including, e.g., [17]. Notice that the second (decoherence) term in (6) can also be rewritten in a standard double-commutator form (see, e.g., [17], [22], [33], and [35]-[39]) since $\left(x-x^{\prime}\right)^{2} \rho\left(x, x^{\prime}\right)=[\hat{x},[\hat{x}, \rho]]_{x, x^{\prime}}$. The nanoresonator evolution described by (6) does not depend on the environment temperature because we essentially assume a large (infinite) quality factor of the nanoresonator, so that the interaction with the thermal bath is much weaker than interaction with the detector, which is infinite by assumption of the effective temperature $T_{d} \sim e V / 2$ [16], [17], [49], [50].

\section{QND SQUEEZING OF THE NANORESONATOR}

\section{A. Modulation of the Measurement Strength}

We assume periodic modulation of the voltage across the QPC detector $V=f(t) V_{0}$, which leads to the corresponding modulation of the parameters in (4) and (6) as follows:

$$
\begin{aligned}
k & =f(t) k_{0} \\
I_{x} & =f(t)\left(I_{00}+k_{0} x\right) \\
S_{I} & =|f(t)| S_{0}
\end{aligned}
$$

while quantum efficiency $\eta$ is assumed to be constant (in the general case, $f(t)$ may become negative). Notice that the noise $\xi(t)$ has an implicit time dependence because of the modulated time spectral density $S_{I}$. The dimensionless coupling is modulated as $\mathcal{C}=|f(t)| \mathcal{C}_{0}$.

In this paper, we will consider two types of modulation with frequency $\omega$ : harmonic modulation with $100 \%$ depth

$$
f(t)=\frac{(1+\cos \omega t)}{2}
$$

and the square-wave (stroboscopic) modulation with pulse width $\delta t$

$$
f(t)=\left\{\begin{array}{l}
1, \quad\left|t-j \times \frac{2 \pi}{\omega}\right| \leq \frac{\delta t}{2}, \quad j=1,2, \ldots \\
0, \quad \text { otherwise. }
\end{array}\right.
$$

Notice that $|f(t)| \leq 1$, so $k_{0}$ and $\mathcal{C}_{0}$ correspond to the maximum coupling. Since $f(t)$ reaches zero in both types of modulation, the conditions $e V \gg \hbar \omega_{0}$ and $k \Delta x_{0} / e \gg \omega_{0}$ required for the Bayesian formalism are violated during a fraction of the modulation period. However, the expected corrections to the Bayesian equations (see, e.g., [17]) have the relative strength of crude $\hbar \omega_{0} / \mathrm{eV}$, which means that the poorly described evolution during these fractions of the period is quite slow. Therefore, we can still use (6) for the analysis in the case of sufficiently large maximum voltage, when the neglected contribution to the evolution during a low-voltage phase is significantly smaller than the well-described contribution during a large-voltage phase. The neglected contribution is expected to lead to a weak relaxation of the nanoresonator state.

\section{B. Simplified Equations for the Gaussian States}

Following [22], [33], [40], and [41], we assume that the oscillator state is Gaussian [42]

$$
\begin{aligned}
\rho\left(x, x^{\prime}\right)= & \frac{1}{\sqrt{2 \pi D_{x}}} \exp \left[-\frac{\left(\frac{x+x^{\prime}}{2}-\langle x\rangle\right)^{2}}{2 D_{x}}\right] \\
& \times \exp \left[-\frac{\left(x-x^{\prime}\right)^{2}}{8 D_{x}} \frac{\left(D_{x} D_{p}-D_{x p}^{2}\right)}{\frac{\hbar^{2}}{4}}\right] \\
& \times \exp \left[i\left(x-x^{\prime}\right)\left(\frac{\langle p\rangle}{\hbar}+\left(\frac{x+x^{\prime}}{2}-\langle x\rangle\right) \frac{D_{x p}}{\hbar D_{x}}\right)\right]
\end{aligned}
$$

and, therefore, is characterized by only five parameters: average position $\langle x\rangle=\langle\hat{x}\rangle$ and momentum $\langle p\rangle=\langle\hat{p}\rangle$, their variances $D_{x}=\left\langle\hat{x}^{2}\right\rangle-\langle\hat{x}\rangle^{2}$ and $D_{p}=\left\langle\hat{p}^{2}\right\rangle-\langle\hat{p}\rangle^{2}$, and the correlation 
$D_{x p}=\langle\hat{x} \hat{p}+\hat{p} \hat{x}\rangle / 2-\langle\hat{x}\rangle\langle\hat{p}\rangle$. These parameters satisfy the generalized Heisenberg inequality [43]

$$
D_{x} D_{p}-D_{x p}^{2} \geq \frac{\hbar^{2}}{4}
$$

which reaches the lower bound for the pure states. The assumption of the Gaussian state can be justified by the fact that a Gaussian state remains Gaussian in the process of continuous measurement [41] (we have checked this statement for nonideal detectors including "asymmetric" detectors and for varying in time strength of measurement) and by the fact that the thermal state (natural initial condition) is Gaussian [42].

For Gaussian states, (6) transforms into

$$
\begin{aligned}
\langle\dot{x}\rangle & =\frac{\langle p\rangle}{m}+\frac{2 k_{0}}{S_{0}} \operatorname{sgn}[f(t)] D_{x} \xi(t) \\
\langle\dot{p}\rangle & =-m \omega_{0}^{2}\langle x\rangle+\frac{2 k_{0}}{S_{0}} \operatorname{sgn}[f(t)] D_{x p} \xi(t)+\mathcal{F} \\
\dot{D}_{x} & =\frac{2}{m} D_{x p}-\frac{2 k_{0}^{2}}{S_{0}}|f(t)| D_{x}^{2} \\
\dot{D}_{p} & =-2 m \omega_{0}^{2} D_{x p}+\frac{k_{0}^{2} \hbar^{2}}{2 S_{0} \eta}|f(t)|-\frac{2 k_{0}^{2}}{S_{0}}|f(t)| D_{x p}^{2} \\
\dot{D}_{x p} & =\frac{1}{m} D_{p}-m \omega_{0}^{2} D_{x}-\frac{2 k_{0}^{2}}{S_{0}}|f(t)| D_{x} D_{x p}
\end{aligned}
$$

which practically coincide with the equations derived in [22], [33], and [40], except for time-dependent $f(t)$. It is interesting to notice that, while (6) is a nonlinear stochastic equation, for which the Stratonovich and Ito forms are significantly different, there is no such difference for (12)-(16), so they can be treated as simple ordinary differential equations.

Notice that the equations for $D_{x}, D_{p}$, and $D_{x p}$ do not depend on noise $\xi(t)$ and feedback force $\mathcal{F}$ and are decoupled from the remaining equations. Therefore, the evolution of the "wave packet width" $\sqrt{D_{x}}$ is deterministic. Analyzing the possibility to squeeze the nanoresonator state, we will consider separately the squeezing of the packet width and the contribution $D_{\langle x\rangle}$ to the total position variance due to fluctuating position of the packet center $\langle x\rangle$. As will be discussed below, $D_{x}$ may be made significantly smaller than the ground state variance $\Delta x_{0}^{2}$ using modulation $f(t)$, while $D_{\langle x\rangle}$ can be made even smaller using the feedback.

\section{Packet Width Squeezing}

Let us use the natural normalization of $D_{x}$ and $D_{p}$ by the ground state parameters, $d_{x} \equiv D_{x} /\left(\hbar / 2 m \omega_{0}\right)$, $d_{p} \equiv D_{p} /\left(\hbar m \omega_{0} / 2\right)$, and similarly $d_{x p} \equiv D_{x p} /(\hbar / 2)$. Then (14)-(16) can be rewritten as

$$
\begin{aligned}
\frac{\dot{d}_{x}}{\omega_{0}} & =2 d_{x p}-\mathcal{C}_{0}|f(t)| d_{x}^{2} \\
\frac{\dot{d}_{p}}{\omega_{0}} & =-2 d_{x p}+\frac{\mathcal{C}_{0}}{\eta}|f(t)|-\mathcal{C}_{0}|f(t)| d_{x p}^{2} \\
\frac{\dot{d}_{x p}}{\omega_{0}} & =d_{p}-d_{x}-\mathcal{C}_{0}|f(t)| d_{x} d_{x p} .
\end{aligned}
$$

We have analyzed these equations numerically for the harmonic (8) and stroboscopic (9) modulation $f(t)$ for several values of the maximum coupling $\mathcal{C}_{0}$, concentrating on the

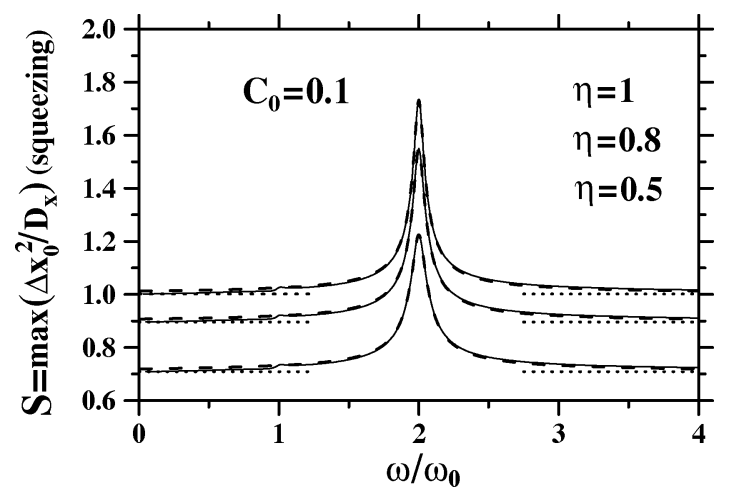

Fig. 1. Dependence of the packet width squeezing $\mathcal{S}$ (maximized over the modulation period) on the frequency $\omega$ of the harmonic modulation (8) of the measurement strength, for three values of the quantum efficiency $\eta$ of the detector. Solid lines show the numerical results, dashed lines are the analytical results corresponding to (25) and (27), and dotted lines are the asymptotes $\mathcal{S}=\sqrt{\eta}$.

range $\mathcal{C}_{0} \leq 1$. Notice that for the stroboscopic modulation the evolution during each period of modulation can be calculated analytically using Riccati equations [33] that significantly simplifies the numerical calculations. As anticipated, we have found that, irrespective of the initial conditions, (17)-(19) approach the asymptotic solutions which oscillate with the modulation frequency $\omega$. Even for small coupling, $\mathcal{C}_{0} \ll 1$, the asymptotic oscillations are significant in the case of resonance: $\omega \simeq 2 \omega_{0}$ for harmonic modulation and $\omega \simeq 2 \omega_{0} / n$ for the stroboscopic modulation (notice that at $\mathcal{C}_{0}=0$ the variances oscillate with frequency $2 \omega_{0}$ ). During the oscillation period, the asymptotic solution for $d_{x}(t)$ reaches the values both above and below the stationary solution for $f(t)=1$ which is [22], [33] $d_{x}=\left(\sqrt{2} / \mathcal{C}_{0}\right)\left[\left(1+\mathcal{C}_{0}^{2} / \eta\right)^{1 / 2}-1\right]^{1 / 2}$ and becomes $d_{x}=1 / \sqrt{\eta}$ for $\mathcal{C}_{0} \ll 1$. Most importantly, the squeezed state $d_{x}<1$ may be achieved for both harmonic and stroboscopic modulation (momentum squeezing is also achieved; however, we do not analyze it in this paper).

Fig. 1 shows the maximum squeezing over the oscillation period for the asymptotic solution, $\mathcal{S}=\max _{t}\left[1 / d_{x}(t)\right]=$ $\max _{t}\left[\Delta x_{0}^{2} / D_{x}(t)\right]$, as a function of the modulation frequency $\omega$ for the harmonic modulation (8) in the case of weak coupling $\mathcal{C}_{0}=0.1$. One can see that, for the ideal detector, $\eta=1$, the squeezing $\mathcal{S} \approx 1.73$ is achieved at $\omega=2 \omega_{0}$ and decreases to $\mathcal{S} \approx 1$ (which corresponds to the ground state width) away from the resonance. The resonances at $\omega=2 \omega_{0} / n, n \geq 2$, are barely visible and lead to small shoulders rather than to peaks. For nonideal detectors, $\eta<1$, the height of the resonance peak decreases, $\mathcal{S}\left(2 \omega_{0}\right) \approx 1.73 \sqrt{\eta}$, while the width increases; the squeezing becomes impossible, $\mathcal{S}<1$, at $\eta<1 / 3$.

Much stronger squeezing of the packet width can be achieved for the stroboscopic modulation (9). (Correspondingly, the efficiency $\eta$ can also be much lower-see analytics below.) Fig. 2 shows $\mathcal{S}(\omega)$ for the ideal detector with $\mathcal{C}_{0}=0.5$ and pulse duration $\delta t=0.05 T_{0}$, where $T_{0}=2 \pi / \omega_{0}$ is the nanoresonator period. One can see that the sharp resonances at $\omega=2 \omega_{0} / n$ have equal height; however, their width decreases with $n$. [If modulation (9) is modified so that $f(t)=$ const $>0$ during the "off" phase, then the peak height also decreases with $n$.] For smaller coupling $\mathcal{C}_{0}$, the peak height remains practically the same, but 


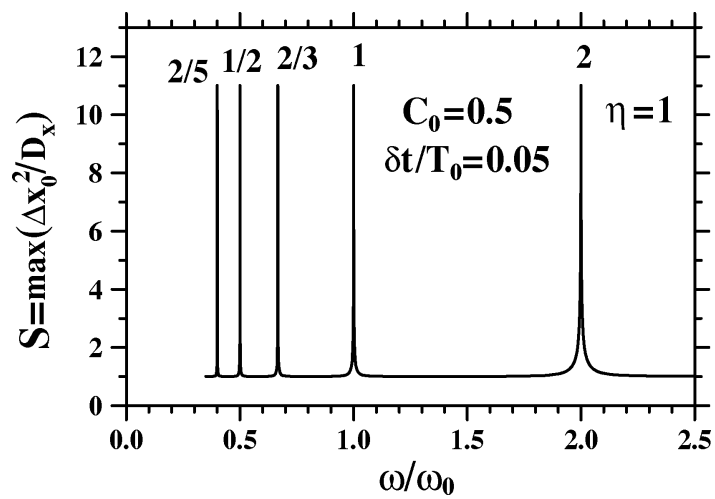

Fig. 2. Numerical results for the packet width squeezing $\mathcal{S}$ as a function of modulation frequency $\omega$ for the stroboscopic measurement modulation (9) with the pulse width $\delta t$. Efficient squeezing occurs at $\omega \approx 2 \omega_{0} / n$. The height of the squeezing peaks is proportional to $\sqrt{\eta}(\delta t)^{-1}$ [see (36)] while their width is proportional to $\mathcal{C}_{0}(\delta t)^{3} / n^{2} \sqrt{\eta}$ [see (37)].

the peak width decreases; this is the reason why we chose relatively large coupling in Fig. 2 in order to have a noticeable peak width. For smaller pulse duration $\delta t$, the squeezing peaks in Fig. 2 would become higher and narrower, while the detector nonideality makes peaks lower and wider (this will be evident from the analytical results discussed below).

1) Evolution of the State Purity: Before discussing the analytical results for squeezing, let us briefly discuss the evolution of the state purity, $\operatorname{Tr}\left(\rho^{2}\right)=(\hbar / 2) / \sqrt{D_{x} D_{p}-D_{x p}^{2}}=1 / \sqrt{u}$, where $u=d_{x} d_{p}-d_{x p}^{2}$. From (17)-(19), it is easy to derive the equation $\dot{u}=\omega_{0} \mathcal{C}_{0}|f(t)| d_{x}\left(\eta^{-1}-u\right)$. Since $\mathcal{C}_{0}$ and $d_{x}$ are positive, the asymptotic solution of this equation is obviously $u=1 / \eta$ and, therefore, the state purity reaches the asymptote $\operatorname{Tr}\left(\rho^{2}\right)=\sqrt{\eta}$. In particular, in the case of ideal detector $\eta=1$, the state eventually becomes pure (similar to the case of a qubit measurement [32], [52]). It is interesting to note that the typical purification time is comparable to the time of reaching the asymptotic regime.

2) Analytics for Harmonic Modulation: Without measurement, $\mathcal{C}_{0}=0$, (17)-(19) have the solution

$$
\begin{aligned}
d_{x}(t) & =\sqrt{\eta^{-1}+A^{2}}-A \cos \left(2 \omega_{0} t+\varphi\right) \\
d_{p}(t) & =\sqrt{\eta^{-1}+A^{2}}+A \cos \left(2 \omega_{0} t+\varphi\right) \\
d_{x p}(t) & =A \sin \left(2 \omega_{0} t+\varphi\right)
\end{aligned}
$$

with arbitrary amplitude $A$ and phase $\varphi$. (Notice that these equations satisfy the condition $u=1 / \eta$.) For weak coupling, $\mathcal{C}_{0} / \eta \ll 1$, and harmonic modulation (8) in the vicinity of the resonance, $\omega \simeq 2 \omega_{0}$, it is natural to look for the asymptotic solution of (17)-(19) in the form of (20)-(22) with $2 \omega_{0}$ replaced with $\omega$ (actually, $A$ and $\varphi$ vary in time with frequency $\omega$, but variations are negligible at $\mathcal{C}_{0} / \eta \ll 1$ ).

To find $A$ and $\varphi$, we substitute these equations into the equation $\int_{-\pi / \omega}^{\pi / \omega} f(t)\left(\eta^{-1}-d_{x}^{2}-d_{x p}^{2}\right) d t=0$ which follows from the stationarity condition $\int_{-\pi / \omega}^{\pi / \omega}\left(\dot{d}_{x}+\dot{d}_{p}\right) d t=0$, and (17) and (18). This gives us the relation

$$
A=\frac{1}{2} \sqrt{\eta^{-1}+A^{2}} \cos \varphi
$$

We find numerically that $\varphi=0$ at the resonance, $\omega=2 \omega_{0}$. (This is quite natural, corresponding to smaller $d_{x}$ at larger measurement strength, and is also proven below). Then, from (23), we find $A=1 / \sqrt{3 \eta}$ and, therefore,

$$
\mathcal{S}\left(2 \omega_{0}\right)=\sqrt{3 \eta}
$$

since the maximum squeezing $\mathcal{S}$ and the amplitude $A$ are related as

$$
\mathcal{S}=\eta\left(A+\sqrt{A^{2}+\eta^{-1}}\right)
$$

This result confirms the numerical result for the peak height in Fig. 1.

To find the shape of the resonant peak, we need one more equation relating $A$ and $\varphi$. It can be obtained by deriving the equation for $\ddot{d}_{x p}(t)$ from (17)-(19), and equating the $\sin (\omega t+\varphi)$ component for the two sides of the equation (assuming $\mathcal{C}_{0} / \eta \ll$ 1 and $\left.\omega \approx 2 \omega_{0}\right)$. In this way, we obtain

$$
\left(4 \omega_{0}^{2}-\omega^{2}\right) A=\eta^{-1} \mathcal{C}_{0} \omega_{0}^{2} \sin \varphi
$$

In particular, this proves that $\varphi=0$ at $\omega=2 \omega_{0}$. Combining (23) and (26), we find the amplitude $A$ as

$$
A(\omega)=\sqrt{\frac{\frac{2}{\eta}}{3+g(\omega)+\sqrt{g^{2}(\omega)+10 g(\omega)+9}}}
$$

where $g(\omega)=16 \eta\left(2-\omega / \omega_{0}\right)^{2} / \mathcal{C}_{0}^{2}$. This result gives us the analytical expression for squeezing $\mathcal{S}$ via (25). The corresponding squeezing is shown by the dashed lines in Fig. 1, which practically coincide with the solid lines representing the numerical results. Notice that the linewidth of the peak is proportional to $\mathcal{C}_{0} / \sqrt{\eta}$; away from the resonance, $A$ decreases to zero, and the squeezing approaches $\mathcal{S}=\sqrt{\eta}$, which is the same as for the case without modulation [33]. The analytical result for $\mathcal{S}(\omega)$ works well for coupling $\mathcal{C}_{0}$ up to approximately 0.3 . It is curious that the rather complex shape of the resonance peak given by (25) and (27) is quite close to the square root of the Lorentzian shape

$$
\mathcal{S}(\omega) \approx \sqrt{\eta}\left(1+\frac{\sqrt{3}-1}{\sqrt{1+\left[\frac{\left(\omega-2 \omega_{0}\right)}{\Delta \omega}\right]^{2}}}\right)
$$

with $\Delta \omega \simeq 0.36 \omega_{0} \mathcal{C}_{0} / \sqrt{\eta}$.

3) Analytics for Stroboscopic Modulation: In the case of stroboscopic modulation (9) of the measurement strength, the variances $d_{x}, d_{p}$, and $d_{x p}$ should follow (20)-(22) during the "off" phase of the modulation, while during the measurement pulse of duration $\delta t$ ("on" phase) the parameters $A$ and $\varphi$ slowly change (we again assume the weak coupling limit) in accordance with (17)-(19). In particular, close to the $n$th resonant peak of Fig. $2, \omega \approx 2 \omega_{0} / n$, the phase $\varphi$ should change during the pulse by the small amount

$$
\delta \varphi=-2 \omega_{0} \frac{2 \pi}{\omega}+2 \pi n \approx \pi n^{2}\left(\frac{\omega}{\omega_{0}}-\frac{2}{n}\right)
$$




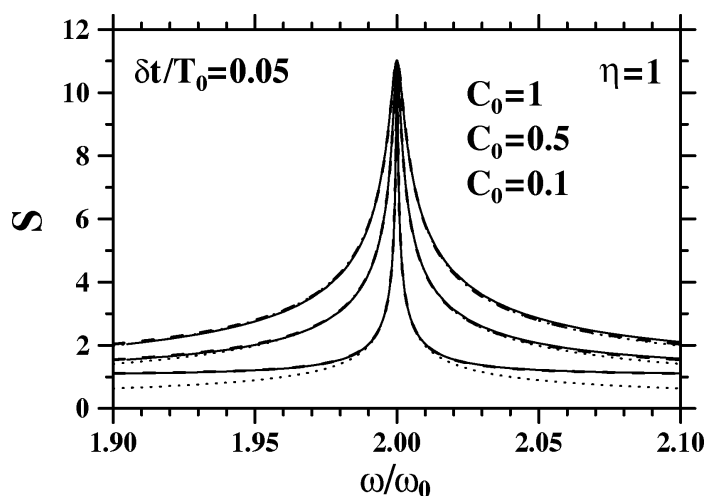

Fig. 3. Squeezing for the stroboscopic modulation for three values of the coupling with detector $\mathcal{C}_{0}$. Solid lines show numerical results, dashed lines (practically coinciding with the solid lines) are the analytical results given by (33) and (25), and the dotted lines are calculated using the simplified equation (35).

in order to match the $2 \pi / \omega$ periodicity of the asymptotic solution with the periodicity of free oscillations (20)-(22). On the other hand, $\delta \varphi$ can be found from the equation

$$
\dot{\varphi}=\frac{-4 \omega_{0} \mathcal{C}_{0} \eta^{-1}|f(t)| d_{x p}}{\left[\left(d_{p}-d_{x}\right)^{2}+4 d_{x p}^{2}\right]}
$$

which follows from from (17)-(19). Integrating (30) within the pulse interval $|t| \leq \delta t / 2$ using (20)-(22) in which $A$ and $\varphi$ are assumed to be constant, we obtain $\delta \varphi=-\mathcal{C}_{0} \sin \left(\omega_{0} \delta t\right) / \eta A$. Combining this result with (29), we obtain an equation relating $A$ and $\varphi$

$$
\pi n^{2} A\left(\frac{\omega}{\omega_{0}}-\frac{2}{n}\right)=\eta^{-1} \mathcal{C}_{0} \sin \left(\omega_{0} \delta t\right) \sin \varphi .
$$

To obtain one more equation for $A$ and $\varphi$, we use the condition $\int_{-\delta t / 2}^{\delta t / 2}\left(\dot{d}_{x}+\dot{d}_{p}\right) d t=0$. Expressing the derivative $\dot{d}_{x}+\dot{d}_{p}$ from (17) and (18) and using (20)-(22), we get the equation

$$
A \omega_{0} \delta t=\sqrt{\eta^{-1}+A^{2}} \sin \left(\omega_{0} \delta t\right) \cos \varphi
$$

Equations (31) and (32) are sufficient to find $A$ for the $n$th resonance, though the expression is quite long as follows:

$$
A^{2}(\omega)=\frac{2 \eta^{-1} \sin ^{2}\left(\omega_{0} \delta t\right)}{B(\omega)+\sqrt{B^{2}(\omega)+4 \tilde{g}(\omega) \sin ^{2}\left(\omega_{0} \delta t\right)}}
$$

where $B(\omega)=\tilde{g}(\omega)+\left(\omega_{0} \delta t\right)^{2}-\sin ^{2}\left(\omega_{0} \delta t\right)$ and $\tilde{g}(\omega)=$ $\pi^{2} n^{2}\left(2 / n-\omega / \omega_{0}\right)^{2} \eta / \mathcal{C}_{0}^{2}$. The squeezing $\mathcal{S}$ is obtained from this result using (25). The corresponding analytical curves are plotted in Fig. 3 by the dashed lines which practically coincide with the numerical results shown by the solid lines. One can see that the analytics work well even for $\mathcal{C}_{0}=1$, even though it was assumed that $\mathcal{C}_{0} \ll 1$ for the derivation.

The value of squeezing at $\omega=2 \omega_{0} / n$ (peak height) can be obtained from (33), but it is easier to use (32) with $\varphi=0$ [which follows from (31)], which leads to the result

$$
\mathcal{S}\left(\frac{2 \omega_{0}}{n}\right)=\sqrt{\eta} \sqrt{\frac{\omega_{0} \delta t+\sin \left(\omega_{0} \delta t\right)}{\omega_{0} \delta t-\sin \left(\omega_{0} \delta t\right)}} .
$$

The analytical results significantly simplify in the case of short pulses, $\delta t \ll T_{0}=2 \pi / \omega_{0}$, then

$$
A^{2}(\omega)=\frac{\frac{6}{\left(\omega_{0} \delta t\right)^{2} \eta}}{1+\sqrt{1+\left[\frac{6 \pi \sqrt{\eta} n^{2}\left(\omega-\frac{2 \omega_{0}}{n}\right)}{\mathcal{C}_{0}(\delta t)^{3} \omega_{0}^{4}}\right]^{2}}}
$$

which corresponds to the peak squeezing

$$
\mathcal{S}\left(\frac{2 \omega_{0}}{n}\right)=\frac{2 \sqrt{3 \eta}}{\omega_{0} \delta t}
$$

and the full width at half height of $\mathcal{S}(\omega)$ equal to

$$
\Delta \omega=\frac{4 \mathcal{C}_{0}(\delta t)^{3} \omega_{0}^{4}}{\pi n^{2} \sqrt{3 \eta}}
$$

The curves calculated using (35) are shown in Fig. 3 by the dotted lines. As one can see, there is a noticeable difference from the numerical results away from the resonance; however, the main part of the peak is fitted quite well.

Equation (36) shows that the maximum squeezing in our model does not depend on coupling $\mathcal{C}_{0}$. Nanoresonator interaction with an extra environment (for example, due to the finite quality factor) would obviously change this conclusion, because the corresponding decay of squeezing would compete with the squeezing "build up," which rate is propotional to $\mathcal{C}_{0}$ as follows from (17)-(19).

\section{Quantum Feedback of the Packet Center}

While the width of the monitored Gaussian packet can be squeezed below the ground state width as shown in the previous subsection, the center of the packet undergoes random evolution described by (12) and (13) and without feedback diffuses far away from the origin. For our model, which assumes an infinite quality factor of the nanoresonator, the packet center evolves infinitely far away because the back-action from the detector "heats up" the nanoresonator to a formally infinite effective temperature (voltage) of the detector [17], [22], [33]. To prevent deviation of the packet center from the origin, we can apply the quantum feedback described by the force $\mathcal{F}$ in (13). Similar to [22] and [33], we choose it as

$$
\mathcal{F}=-m \omega_{0} \gamma_{x}\langle x\rangle-\gamma_{p}\langle p\rangle
$$

Notice that the random oscillating evolution of $\langle x\rangle$ and $\langle p\rangle$ can be extracted from the measurement record using (12) and (13) even when the measurement is performed during only small fraction of the period.

Following [22] and [33], we characterize the distribution of the packet center position $\langle x\rangle$ and center momentum $\langle p\rangle$ by the ensemble averages (over realizations) $\langle\langle x\rangle\rangle$ and $\langle\langle p\rangle\rangle$ and the variances $D_{\langle x\rangle}=\left\langle\langle x\rangle^{2}\right\rangle-\langle\langle x\rangle\rangle^{2}, D_{\langle p\rangle}=\left\langle\langle p\rangle^{2}\right\rangle-\langle\langle p\rangle\rangle^{2}$, and $D_{\langle x\rangle\langle p\rangle}=\langle\langle x\rangle\langle p\rangle\rangle-\langle\langle x\rangle\rangle\langle\langle p\rangle\rangle$. In the notation of doubled angle brackets, the inner brackets mean averaging with the density matrix $\rho$ in an individual realization of the process, while the outer brackets represent averaging over realizations. 
The equations for $\langle\langle\dot{x}\rangle\rangle$ and $\langle\langle\dot{p}\rangle\rangle$ derived from (12) and (13) lead to the ensemble averaged position evolution as $\langle\langle\ddot{x}\rangle\rangle+$ $\gamma_{p}\langle\langle\dot{x}\rangle\rangle+\left(\omega_{0}^{2}+\gamma_{x} \omega_{0}\right)\langle\langle x\rangle\rangle=0$, from which it is clear that $\langle\langle x\rangle\rangle$ relaxes to zero for positive $\gamma_{p}$.

Introducing dimensionless variances $d_{\langle x\rangle} \equiv D_{\langle x\rangle} 2 m \omega_{0} / \hbar$, $d_{\langle p\rangle} \equiv D_{\langle p\rangle} 2 / \hbar m \omega_{0}$, and $d_{\langle x\rangle\langle p\rangle} \equiv D_{\langle x\rangle\langle p\rangle} 2 / \hbar$, we derive the following equations from (12) and (13):

$$
\begin{aligned}
& \frac{\dot{d}_{\langle x\rangle}}{\omega_{0}}=2 d_{\langle x\rangle\langle p\rangle}+\mathcal{C}_{0}|f(t)| d_{x}^{2} \\
& \frac{\dot{d}_{\langle p\rangle}}{\omega_{0}}=-2 d_{\langle x\rangle\langle p\rangle}-2 \mu F d_{\langle x\rangle\langle p\rangle}-2 F d_{\langle p\rangle}+\mathcal{C}_{0}|f(t)| d_{x p}^{2}
\end{aligned}
$$

$\frac{\dot{d}_{\langle x\rangle\langle p\rangle}}{\omega_{0}}=d_{\langle p\rangle}-d_{\langle x\rangle}-\mu F d_{\langle x\rangle}-F d_{\langle x\rangle\langle p\rangle}+\mathcal{C}_{0}|f(t)| d_{x} d_{x p}$

where $F=\gamma_{p} / \omega_{0}$ and $\mu=\gamma_{x} / \gamma_{p}$ are the dimensionless feedback parameters.

We have simulated these equations numerically using the asymptotic solutions of (17)-(19) for $d_{x}, d_{p}$, and $d_{x p}$. We have mostly studied the resonance $\omega=2 \omega_{0}$ in the weakly coupling regime. The main finding is that, for both harmonic [see (8)] and stroboscopic [see (9)] modulation of measurement, the center position variance $d_{\langle x\rangle}$ can be made much smaller than the packet variance $d_{x}$ at time moments $t=j 2 \pi / \omega$ when the packet squeezing is at a maximum. Therefore, the corresponding worsening of the ensemble-averaged squeezing defined as $\left(d_{x}+d_{\langle x\rangle}+\langle\langle x\rangle\rangle^{2} / \Delta x_{0}^{2}\right)^{-1}$ is negligible.

As an example, for the stroboscopic modulation with $\delta t / T_{0}=0.05$ and $\mathcal{C}_{0}=0.1$ at $\omega=2 \omega_{0}$, the ratio $d_{\langle x\rangle} / d_{x}$ at $t=j T_{0} / 2$ is around $0.4 \%$ for $\mu=0$ and $F=0.5$ and around $0.1 \%$ for $\mu=10$ and $F \gg 1$. [The term with $\gamma_{x}$ in (38) is not necessary; however, it improves squeezing of the packet center, so nonzero $\mu$ is beneficial.] The ratio $d_{\langle x\rangle} / d_{x}$ decreases with a decrease of the pulse width $\delta t$ and a decrease of coupling $\mathcal{C}_{0}$. It is important to notice that $d_{\langle x\rangle}$ scales linearly with $\mathcal{C}_{0}$ as follows from (39)-(41), while $d_{x}$ as well as $d_{p}$ and $d_{x p}$ do not depend on $\mathcal{C}_{0}$ at $\omega=2 \omega_{0}$ and $\mathcal{C}_{0} / \eta \ll 1$ (this was also checked numerically). Therefore, the ratio $d_{\langle x\rangle} / d_{x}$ can be made arbitrary small at small coupling.

The analytical results (which will be described in more detail elsewhere) show that, in the case $\mu \omega_{0} \delta t \gg 1$ and $F \gg 1$, the variance of the packet center at the middle of the measurement pulse is $d_{\langle x\rangle}=\mathcal{C}_{0}\left(\omega_{0} \delta t\right)^{2} / 24 \mu \eta$, which can obviously be made much smaller than $\mathcal{S}^{-1}$ given by (36) at sufficiently small $\mathcal{C}_{0} / \sqrt{\eta}$ and $\omega_{0} \delta t$ and/or sufficiently large $\mu$.

\section{E. Observability of the Squeezed State}

The fact that the squeezed state of a nanoresonator can be prepared by the modulated measurement and quantum feedback does not automatically mean that this state may be useful for the measurement of extremely weak forces and even that such state can be checked experimentally in a straightforward way. As an example of such a problem, in one of the setups analyzed in [44] the squeezed in-loop optical state is realized by using quantum feedback, but the squeezing of the output light is impossible. Fortunately, as we discuss below, in our case there is no problem with observability of the squeezed state.
We have studied the possibility to verify the squeezed state of the nanoresonator in the following way. After the preparation of the squeezed state by stroboscopic measurement and feedback, the feedback at some moment $(t=0)$ is switched off, while the stroboscopic measurement continues. Considering for simplicity the case of one measurement per nanoresonator period ( $n=2, \omega=\omega_{0}$ ), we calculate the average of the position measurements (each pulse gives a very imprecise measurement because of weak coupling)

$$
X_{N}=\frac{1}{N} \sum_{j=1}^{N} \frac{1}{\delta t k_{0}} \int_{j T_{0}-\frac{\delta t}{2}}^{j T_{0}+\frac{\delta t}{2}}\left[I(t)-I_{00}\right] d t .
$$

The idea is that, for a squeezed initial state, $X_{N}$ can be much closer to zero than if we would start with the ground state.

The analysis of the distribution of $X_{N}$ (over realizations) is very simple in the case of instantaneous but imprecise measurements, $\delta t \rightarrow 0, \mathcal{C}_{0} \delta t=$ const, since the Hamiltonian evolution of the resonator in between the measurements can be completely neglected and, therefore, $N$ measurements are equivalent to one $\mathrm{N}$-times stronger measurement. This gives us the variance of $X_{N}$ equal to

$$
D_{X, N}=\frac{\hbar}{2 m \omega_{0}}\left(\frac{1}{\mathcal{S}}+\frac{1}{N \mathcal{C}_{0} \omega_{0} \delta t}\right)
$$

where the first term is due to the initial packet width, while the second term is the inaccuracy of the measurement which improves with $N$. Obviously, at $N \gg 1 / \mathcal{C}_{0} \omega_{0} \delta t$, this variance for a squeezed state $(\mathcal{S}>1)$ is significantly smaller than the variance for the ground state $(\mathcal{S}=1)$. Even though this difference can be rigorously verified only by performing many experiments to accumulate statistics for $D_{X, N}$, it can be observed even in a single experiment with good reliability if $\mathcal{S} \gg 1$. (In a single realization, the failure probability for distinguishing squeezed and ground states is crudely $\mathcal{S}^{-1 / 2}$.)

Unfortunately, this result requires the assumption of infinitely strong coupling, so it is not obvious if it holds in the case of weak coupling or not. The possible problem is that for sufficiently large $N$ which makes the second term in (43) sufficiently small, the nanoresonator heating due to measurement back-action may already eliminate the squeezing (the feedback is off). We have calculated $D_{X, N}$ for stroboscopic modulation numerically using (12) and (13) and have found that there is still a range of $N$ where the squeezed and ground initial states lead to significantly different $D_{X, N}$ and, therefore, can be reliably distinguished. As an example, for $\mathcal{C}_{0}=0.1, \eta=1$, and $\delta t=0.02 T_{0}$, the normalized variance $d_{X, N} \equiv D_{X, N} 2 m \omega_{0} / \hbar$ achieves a minimum of 0.078 (at $N \simeq 4 \times 10^{3}$ ), which is crudely two times larger than the contribution from the initial squeezing $1 / \mathcal{S}=0.036$ and is still significantly smaller than the ground state limit $d_{X, N} \geq 1$. We have found numerically that the minimum $d_{X, N}$ scales linearly with the pulse width $\delta t$ (similarly to $1 / \mathcal{S}$ ) and practically does not depend on coupling $\mathcal{C}_{0}$ at $\mathcal{C}_{0} \leq 1$. This hints that the product $\mathcal{S} \times \min _{N} d_{X, N}$ is practically a constant approximately equal to 2 and, therefore, verification of the squeezed state by a weakly coupled detector is almost as efficient as the verification by instantaneous measurements (within a factor of about 2). 


\section{CONCLUSION}

In this paper, we have shown that the uncertainty of the nanoresonator position can be squeezed significantly below the ground state level by the modulated in time measurement of the nanoresonator position with the QPC or RF-SET detector. The measurement strength is modulated by applying the periodic voltage across the detector. The mechanism of squeezing is similar to the QND measurements [1] (cancelled Hamiltonian evolution allows the buildup of the effective measurement strength for repeated weak measurements), though a significant difference in our case is the continuous measurement with weak coupling to the detector. We have considered harmonic [see (8)] and stroboscopic [see (9)] modulations and found that only a moderate squeezing $\mathcal{S} \leq \sqrt{3 \eta}$ is possible for the harmonic modulation with frequency $\omega \approx 2 \omega_{0}$ [see (24), (27), and Fig. 1]. However, the stroboscopic modulation can lead to an arbitrary strong squeezing $\mathcal{S} \leq 2 \sqrt{3 \eta} / \omega_{0} \delta t$ for sufficiently short measurement pulses $\delta t$ applied with frequency $\omega=2 \omega_{0} / n$ [see (34)-(37) and Fig. 2]. Obviously, the state width oscillates with time, so that the maximum position squeezing is achieved periodically (with a period close to $\pi / \omega_{0}$ ), while the maximum squeezing of momentum happens with $\pi / 2 \omega_{0}=T_{0} / 4$ time shift (when $\mathcal{S}<1$ ).

While the modulated measurement squeezes the width of the state (packet), the position of the packet center $\langle x\rangle$ fluctuates due to random back-action from the detector; so, to keep the packet center near $x=0$, we need to apply quantum feedback. We have found that the feedback can keep the deviation of $\langle x\rangle$ from zero much smaller than the packet width, which means that the ensemble-averaged squeezing practically does not differ from the packet width squeezing.

In this paper, we have used the Bayesian formalism [32], [52] for the description of the quantum measurement process. However, since the obtained equations practically coincide with the equations used in [22] and [33], we have followed those papers to a large extent, especially for the analysis of the evolution of the Gaussian states.

An important issue is the possibility to use the squeezed states of the nanoresonator for the measurement of weak forces with the accuracy beyond the standard quantum limit. Even though we did not consider this question explicitly, we have found that the state squeezing can be verified (with high reliability) even in a single measurement run by a weakly coupled detector.

The main drawback of the present theory is the assumption of a very large quality factor $Q$ of the nanoresonator. Crude preliminary analysis indicates that our results for the stroboscopic modulation are valid for $Q \gg \mathcal{S}^{3} / \mathcal{C}_{0} \sqrt{\eta} \sim \eta / \mathcal{C}_{0}\left(\omega_{0} \delta t\right)^{3}$ and sufficiently small temperature of the environment, $T / \hbar \omega_{0} \ll \sqrt{\eta} Q \mathcal{C}_{0} / \mathcal{S}^{3} \sim Q \mathcal{C}_{0}\left(\omega_{0} \delta t\right)^{3} / \eta$. These conditions seem to be within present-day experimental reach for moderate squeezing $\mathcal{S}$.

\section{ACKNOWLEDGMENT}

The authors would like to thank D. Averin, A. Doherty, S. Habib, K. Jacobs, K. Likharev, I. Martin, and G. Milburn for fruitful discussions and remarks.

\section{REFERENCES}

[1] V. B. Braginsky and F. Y. Khalili, Quantum Measurement. Cambridge, U.K.: Cambridge Univ. Press, 1992.

[2] V. B. Braginsky, Y. I. Vorontsov, and F. Y. Khalili, "Optimal quantum measurements in detectors of gravitation radiation," JETP Lett., vol. 27, pp. 276-280, 1978.

[3] K. S. Thorne, R. W. P. Drever, C. M. Caves, M. Zimmermann, and V. D. Sandberg, "Quantum nondemolition measurements of harmonic oscillators," Phys. Rev. Lett., vol. 40, pp. 667-671, 1978.

[4] C. M. Caves, K. S. Thorne, R. W. P. Drever, V. D. Sandberg, and M. Zimmermann, "On the measurement of a weak classical force coupled to a quantum-mechanical oscillator. I. Issues of principle," Rev. Mod. Phys., vol. 52, pp. 341-392, 1980.

[5] H. J. Kimble, Y. Levin, A. B. Matsko, K. S. Thorne, and S. P. Vyatchanin, "Conversion of conventional gravitational-wave interferometers into quantum nondemolition interferometers by modifying their input and/or output optics," Phys. Rev. D, Part. Fields, vol. 65, pp. 022 002-1-022 002-31, 2002.

[6] V. B. Braginskii, "Adolescent years of experimental physics," PhysicsUspekhi, vol. 46, pp. 81-87, 2003.

[7] D. V. Averin, "Quantum nondemolition measurements of a qubit," Phys. Rev. Lett., vol. 88, pp. 207 901-1-207901-4, 2002.

[8] L. Bulaevskii, M. Hruska, A. Shnirman, D. Smith, and Y. Makhlin, "Nondemolition measurements of a single quantum spin using Josephson oscillations," Phys. Rev. Lett., vol. 92, p. 177 001, 2004.

[9] J. M. Geremia, J. K. Stockton, and H. Mabuchi, "Real-time quantum feedback control of atomic spin-squeezing," Science, vol. 304, pp. 270-273, 2004.

[10] A. N. Cleland and M. L. Roukes, "Fabrication of high frequency nanometer scale mechanical resonators from bulk Si crystals," Appl. Phys. Lett., vol. 69, pp. 2653-2655, 1996.

[11] D. W. Carr and H. G. Craighead, "Fabrication of nanoelectromechanical systems in single crystal silicon using silicon on insulator substrates and electron beam lithography," J. Vacuum Sci. Technol. B, Microelectron. Process. Phenom., vol. 15, pp. 2760-2763, 1997.

[12] X. Ming, H. Huang, C. A. Zorman, M. Mehregany, and M. L. Roukes, "Nanodevice motion at microwave frequencies," Nature, vol. 421, p. 496, 2003.

[13] R. G. Knobel and A. N. Cleland, "Nanometer-scale displacement sensing using a single electron transistor," Nature, vol. 424, pp. 291-293, 2003.

[14] M. D. LaHaye, O. Buu, B. Camarota, and K. C. Schwab, “Approaching the quantum limit of a nanomechanical resonator," Science, vol. 304, pp. 74-77, 2004.

[15] A. N. Cleland, J. S. Aldridge, D. C. Driscoll, and A. C. Gossard, "Nanomechanical displacement sensing using a quantum point contact," Appl. Phys. Lett., vol. 81, pp. 1699-1701, 2002.

[16] M. Blencowe, "Quantum electromechanical systems," Phys. Reports, vol. 395, pp. 159-222, 2004.

[17] D. Mozyrsky and I. Martin, "Quantum-classical transition induced by electrical measurement," Phys. Rev. Lett., vol. 89, pp. 018 301-1-018301-4, 2002.

[18] A. D. Armour, M. P. Blencowe, and K. C. Schwab, "Entanglement and decoherence of a micromechanical resonator via coupling to a Cooperpair box," Phys. Rev. Lett., vol. 88, pp. 148 301-1-148 301-4, 2002.

[19] A. Y. Smirnov, L. G. Mourokh, and N. J. M. Horing, "Nonequilibrium fluctuations and decoherence in nanomechanical devices coupled to the tunnel junction," Phys. Rev. B, Condens. Matter, vol. 67, pp. 115312 1-115 312-9, 2003.

[20] K. Schwab, "Spring constant and damping constant tuning of nanomechanical resonators using a single-electron transistor," Appl. Phys. Lett., vol. 80, pp. 1276-1278, 2002.

[21] R. Knobel and A. N. Cleland, "Piezoelectric displacement sensing with a single-electron transistor," Appl. Phys. Lett., vol. 81, pp. 2258-2260, 2002.

[22] A. Hopkins, K. Jacobs, S. Habib, and K. Schwab, "Feedback cooling of a nanomechanical resonator," Phys. Rev. B, Condens. Matter, vol. 68, pp. $235328-1-235328-10,2003$.

[23] I. Martin, A. Shnirman, L. Tian, and P. Zoller, "Ground state cooling of mechanical resonators," Phys. Rev. B, Condens. Matter, vol. 69, pp. 125 339-1-125 339-12, 2004.

[24] R. Ruskov and A. N. Korotkov, "Quantum feedback control of a solid-state qubit," Phys. Rev. B, Condens. Matter, vol. 66, pp. 041 401-1-041 401-4, 2002.

[25] H. M. Wiseman and G. J. Milburn, "Quantum-theory of optical feedback via homodyne detection,” Phys. Rev. Lett., vol. 70, pp. 548-551, 1993. 
[26] M. P. Blencowe and M. N. Wybourne, "Quantum squeezing of mechanical motion for micron-sized cantilevers," Physica B, vol. 280, pp. 555-556, 2000.

[27] D. Rugar and P. Grütter, "Mechanical parametric amplification and thermomechanical noise squeezing," Phys. Rev. Lett., vol. 67, pp. 699-702, 1991.

[28] S. A. Gurvitz, "Measurements with a noninvasive detector and dephasing mechanism," Phys. Rev. B, Condens. Matter, vol. 56, pp. $15215-15223,1997$.

[29] A. N. Korotkov and D. V. Averin, "Continuous weak measurement of quantum coherent oscillations," Phys. Rev. B, Condens. Matter, vol. 61, p. 165310,2001

[30] H. S. Goan and G. J. Milburn, "Dynamics of a mesoscopic charge quantum bit under continuous quantum measurement," Phys. Rev. B, Condens. Matter, vol. 64, pp. 235 307-1-235 307-12, 2001.

[31] A. N. Korotkov, "Nonideal quantum detectors in Bayesian formalism," Phys. Rev. B, Condens. Matter, vol. 67, pp. 235 408-1-235 408-11, 2003.

[32] - "Continuous quantum measurement of a double dot," Phys. Rev. B, Condens. Matter, vol. 60, pp. 5737-5741, 1999.

[33] A. C. Doherty and K. Jacobs, "Feedback control of quantum systems using continuous state estimation," Phys. Rev. A, Gen. Phys., vol. 60, pp. 2700-2711, 1999.

[34] M. B. Mensky, "Decoherence and the theory of continuous quantum measurements," Physics-Uspekhi, vol. 168, pp. 1017-1035, 1998.

[35] M. J. Gagen, H. M. Wiseman, and G. J. Milburn, Phys. Rev. A, Gen. Phys., vol. 48, pp. 132-142, 1993.

[36] G. Lindblad, "Generators of quantum dynamical semigroups," Comm. Math. Phys., vol. 48, pp. 119-130, 1976.

[37] A. O. Caldeira and A. J. Leggett, "Quantum tunnelling in a dissipative system," Ann. Phys. (N.Y.), vol. 149, pp. 374-456, 1983.

[38] C. Caves and G. J. Milburn, "Quantum-mechanical model for continuous position measurements," Phys. Rev. A, Gen. Phys., vol. 36, pp. 5543-5555, 1987.

[39] W. H. Zurek, S. Habib, and J. P. Paz, "Coherent states via decoherence," Phys. Rev. Lett., vol. 70, pp. 1187-1190, 1993.

[40] J. Halliwell and A. Zoupas, "Quantum state diffusion, density matrix diagonalization, and decoherent histories: a model," Phys. Rev. D, Part. Fields, vol. 52, pp. 7294-7307, 1995.

[41] J. K. Breslin and G. J. Milburn, "Conditional variance reduction by measurements on correlated field modes," Phys. Rev. A, Gen. Phys., vol. 55, pp. 1430-1436, 1997.

[42] C. W. Gardiner, Quantum Noise. Berlin, Germany: Springer-Verlag, 1991.

[43] V. V. Dodonov, E. V. Kurmyshev, and V. I. Man'ko, "Generalized uncertainty relation and correlated coherent states," Phys. Lett. A, vol. 79, pp. 150-152, 1980.

[44] H. M. Wiseman and G. J. Milburn, "Squeezing via feedback," Phys. Rev. A, Gen. Phys., vol. 49, pp. 1350-1366, 1994.

[45] A. N. Cleland and M. L. Roukes, "A nanometer-scale mechanical electrometer," Nature, vol. 392, pp. 160-162, 1998.
[46] E. Buks and M. L. Roukes, "Metastability and the Casimir effect in micromechanical systems," Europhys. Lett., vol. 54, pp. 220-226, 2001.

[47] H. G. Craighead, "Nanoelectromechanical systems," Science, vol. 290, pp. $1532-1535,2000$

[48] M. Zalalutdinov, B. Ilic, D. Czaplewski, A. Zehnder, H. G. Craighead, and J. M. Parpia, "Frequency-tunable micromechanical oscillator," Appl. Phys. Lett., vol. 77, pp. 3287-3289, 2000.

[49] M. P. Blencowe and M. N. Wybourne, "Sensitivity of a micromechanical displacement detector based on the radio-frequency single-electron transistor," Appl. Phys. Lett., vol. 77, pp. 3845-3847, 2000.

[50] A. D. Armour, M. P. Blencowe, and Y. Zhang, "Classical dynamics of a nanomechanical resonator coupled to a single-electron transistor," Phys. Rev. B, Condens. Matter, vol. 69, pp. 125 313-1-125 313-15, 2004.

[51] A. N. Korotkov, "Simple Quantum Feedback of a Solid-State Qubit,", cond-mat/0404696, 2004

[52] - "Selective evolution of a qubit state due to continuous measurement," Phys. Rev. B, Condens. Matter, vol. 63, p. 115 403, 2001.

Rusko Ruskov received the Ph.D. degree from the Joint Institute for Nuclear Research (JINR), Dubna, Russia, in 1996.

He has been with JINR and the Institute for Nuclear Research and Nuclear Energy, Sofia, Bulgaria. He is currently with the University of California, Riverside. His current research interest includes continuous quantum measurement of solid-state systems (qubits and nanoresonators) and quantum feedback control.

Keith Schwab received the Ph.D. degree from the University of California at Berkeley (UC Berkeley), in 1996.

While with UC Berkeley, he developed and demonstrated a superfluid SQUID device. He then demonstrated quantized thermal transport in a nanodevice at the California Institute of Technology, Pasadena, in 1999, where he was a Post-Doctoral Scholar. In 2000, he joined the National Security Agency's Laboratory for Physical Sciences, College Park, MD, where he leads an ultralow-temperature group focused on nanomechanics, qubits, and quantum measurement. He has authored over 30 journal papers.

Alexander N. Korotkov received the Ph.D. degree from Moscow State University, Moscow, Russia, in 1991.

After that, he was with Moscow State University and with Stony Brook University, Stony Brook, NY. In 2000, he joined the faculty of the University of California, Riverside, where he is currently an Associate Professor. His research interests include single electronics (physics and device applications), noise analysis, and quantum measurements in mesoscopic systems. He is the author/coauthor of over 70 journal papers which have been cited over 1000 times. 